predictive factor for mortality $(p=0.02), H R=3.2$ (1.3 to 7.7), adjusted for the other scales, gender and age (Cox).

Discussion and Conclusions Of the elders' morbidity, ADL alterations are the causes for most concern as they are already noted to be predictive for mortality after 3 years of the cohort's survival.

\section{SP3-42 TRENDS AND CHARACTERISTICS OF SELF-REPORTED HIV TESTING IN WOMEN OF CHILDBEARING AGE, IN PERU 2000, 2004-2008}

doi:10.1136/jech.2011.1429760.42

\begin{abstract}
1,2R Tejada,* ${ }^{1} \mathrm{~J}$ Alarcón, ${ }^{3} \mathrm{~J}$ Zunt, ${ }^{4} \mathrm{~S}$ Montano. ${ }^{1}$ Universidad Nacional Mayor de San Marcos, Lima, Lima, Peru; ${ }^{2}$ Fogarty International Center, Bethesda, Maryland, USA; ${ }^{3}$ University of Washington, Seattle, Washington, USA; ${ }^{4}$ US Naval Medical Search Center Detachment, Lima, Lima, Peru
\end{abstract}

Introduction In the 26-year HIV/AIDS epidemic in Peru more than 39682 cases of HIV and 25533 cases of AIDS have been reported. Despite a higher concentration of the epidemic in men who have sex with men, there has been transmission to other groups and a decline in the male to female ratio of HIV infection. The objective of our study was to examine trends and factors associated with obtaining HIV testing for women of childbearing age (WCBA).

Methods We used Demographic and Family Health Surveys databases of Peru from the years 2000, and 2004 to 2008 to study trends of self-reported HIV testing. The association between HIV testing and sociodemographic and reproductive health factors was assessed using bivariate analyses and logistic regression.

Results We analysed data from 124797 Peruvian WCBA. There was a significant difference in the number of WCBA who reported having been tested for HIV, with more than a doubling of testing between $2000(15.3 \%)$ and $2008(39.6 \%)(p<0.001)$. HIV testing was associated with urban residence (OR 1.96), higher educational level (OR 5.14), speaking Spanish (OR 3.10), having a partner (OR 3.21), self-perception of high risk for HIV (OR 1.41), and having had a sexually transmitted infection in the preceding year (OR 2.18).

Conclusion There has been an increasing number of WCBA who report HIV testing since 2000, but less than half of WCBA do not seek HIV testing. Major factors associated with HIV testing should be considered when planning new initiatives to increase HIV testing of WCBA.

\section{SP3-43 FACTORS INFLUENCING WOMEN TO RETURN TO RECEIVE THEIR PAP SMEAR RESULTS, IN THE STATE CAPITAL OF RIO DE JANEIRO IN BRAZIL}

doi:10.1136/jech.2011.1429760.43

L de Almeida, ${ }^{*}$ L F Martins, M Souza, E Fernandes, E Moraes. Instituto Nacional de Câncer, Rio de Janeiro, Brazil

Introduction Although actions for cervical cancer reduction in primary care were implemented in Rio de Janeiro in the '80s, the incidence and associated mortality are still among the highest in the country. For effective prevention it is essential women complete follow-up after their Pap smear. More information about the reasons preventing follow-up is needed.

Objective To investigate the factors that influence women not to return to receive their Pap smear result in the Brazilian Public Health System in Rio de Janeiro city, Brazil.

Material and Methods A cross-sectional study was conducted. All participants signed a consent form and 1278 women completed the protocol. The questionnaire included: socio-demographic status, self-evaluation about health status, knowledge about prevention, last Pap smear and reproductive history.
Results The percentage of women who did not return to receive the result of Pap smear was 23.1\% (18.3-27.9). Schooling, marital status, race, religion and per capita income, self-evaluation about health status, knowledge about prevention, reason to perform exam, satisfaction level in medical attendance were not associated with the return for getting the results. Women who were not informed of a date to return for heir result were less likely to return than those who had been informed, 47.0\% (35.9-58.4).

Discussion The results showed that the behaviour of the health professional and the infrastructure of the healthcare system can influence the return of women to receive the Pap smear result and their follow-up treatment.

\section{SP3-44 PREGNANCY IN ADOLESCENCE: ALWAYS UNWANTED?}

doi:10.1136/jech.2011.142976o.44

${ }^{1,2} \mathrm{C}$ Meneses, ${ }^{*} \mathrm{C}$ Lopes, ${ }^{1,3} \mathrm{~V}$ Magalhães. ${ }^{1}$ UERJ, Rio de Janeiro, Brazil; ${ }^{2}$ UNIGRANRIO, Rio de Janeiro, Brazil; ${ }^{3}$ CELSO LISBOA, Rio de Janeiro, Brazil

Introduction Studies conducted all over the world show that the rates of pregnancy in adolescence are increasing, especially in the early adolescence (10-14 years of age). Although the majority of pregnancies in this period are not desired some studies suggest that a number of them are wanted. Aims: (1) Assess the prevalence of wanted pregnancy and possible associations with socioeconomic, demographic factors and social support network in a population of pregnant adolescents (2) Verify if age should be an effect modifier in these associations.

Methods Cross sectional study conducted with pregnant adolescents regularly attending two public maternity units. A sample of 232 pregnant adolescents aged 13-20 years (mean 17.3 years) completed a self-reported questionnaire.

Results Prevalence of intended pregnancy was $46.2 \%$. Final adjusted model showed that being married (PR 1.80, 95\% CI 1.27 to 2.56 ) and not having friends (PR 1.48, 95\% CI 1.15 to 1.90) showed association with the desire to be pregnant. Girls studying in the elementary grade and aged $12-16$ years desired to be pregnant less (PR 0.57, 95\% CI 0.38 to 0.88 ).

Conclusion Pregnancy in adolescence may not always be unwanted. Some factors, such as being married and not having any friends may have influence in these cases. Professionals dealing with adolescents should be aware of these issues in order to identify high risk situations that could be managed.

\section{SP3-45 AIDS-FREE-TIME IN THE PRE AND POST-HAART ERAS IN THE SAO PAULO HIV COHORT, BRAZIL}

doi:10.1136/jech.2011.1429760.45

${ }^{1} \mathrm{M}$ V Tancredi, ${ }^{2} \mathrm{E}$ A Waldman. ${ }^{1}$ Sao Paulo State STD and AIDS Training and Reference Center, Sao Paulo, Sao Paulo, Brazil; ${ }^{2}$ School of Public Health of Sao Paulo University, Sao Paulo, Sao Paulo, Brazil

Background The effect of ART used before aids diagnosis has been the object of studies. The objectives were to estimate AIDS incidence rates, median AIDS-free-time and to investigate predictor factors for progression to AIDS

Methods Retrospective cohort study, encompassing 1879 adult patients of the HIV São Paulo Cohort, during 1988-2005. The Kaplan-Meier methods, the Cox proportional hazard model and HRs estimates were used.

Results 981 patients progressed to aids. AIDS incidence rates were 11.6 and 7.1 person-years in the 1988-1996 and 1997-2003 periods, respectively. The median time of progression from HIV infection to AIDS without treatment was 53.7 months; with ART without HAART, 90.0 months; and with HAART, over $50 \%$ of patients 
followed did not progress to AIDS until 108 months. Independent prognostic factors for AIDS-free-time were: treatment with ART without HAART (HR 2.1; 95\% CI 1.6 to 2.8), no treatment regimen (HR 3.0; 95\% CI 2.5 to 3.6); age at HIV infection diagnosis between 30 and 49 years (HR 1.2; 95\% CI 1.1 to 1.3 ), age over 50 years (HR 2.9; $95 \%$ CI 2.3 to 5.2 ); black race/colour (HR 1.4; $95 \%$ CI 1.1 to 1.7 ); MSM (HR 1.4; 95\% CI 1.1 to 1.6) and IDU (HR 1.7; 95\% CI 1.3 to 2.2) exposure categories; up to 8 years of schooling (HR 1.3; $95 \% \mathrm{CI}$ 1.1 to 1.5 ) and no schooling (HR 2.0; 95\% CI 1.4 to 5.6); and CD4 count between 350 and 500 cells $/ \mathrm{mm}^{3}$ (HR 1.6; 95\% CI 1.3 to 1.9). Conclusions Increased AIDS-free-time was observed, with HAART. Decrease in the incidence rates were observed, Predictor factors to AIDS were treatment, age, race/colour, transmission categories, schooling and CD4 count.

\section{SP3-46 AIDS SURVIVAL IN THE PRE AND POST-HAART ERAS IN THE SAO PAULO AIDS COHORT, BRAZIL}

doi:10.1136/jech.2011.1429760.46

${ }^{1} \mathrm{M}$ V Tancredi, ${ }^{2} E$ A Waldman. ${ }^{1}$ Sao Paulo State STD and AIDS Training and Reference Center, Sao Paulo, Sao Paulo, Brazil; ' 2 School of Public Health of Sao Paulo University, Sao Paulo, Sao Paulo, Brazil

Background AIDS remains a great public health problem and the effect of ART has been studied. The objectives were to estimate AIDS mortality rates, median survival time and to investigate death predictor factors.

Methods Retrospective cohort study, with 6594 adult patients followed from 1988 to 2005. The Kaplan-Meier estimator, the Cox proportional hazard model and HRs estimates were used.

Results In a follow-up of 203008 persons-year, 2936 patients progressed to death. AIDS mortality rates were 17.6, 23.2, and 7.8 person-years in the 1988-1993, 1994-1996 and 1997-2003 periods, respectively. Median progression time from AIDS to death was 13.4 months in the 1988-1993 period; 22.3 months, between 1994 and 1996, and in the 1997-2003 period, over 50\% of patients followed survived. Independent predictor factors for death were: AIDS diagnosis period 1994-1996 (HR 2.0; 95\% CI 1.8 to 2.2) and 1988-1993 (HR 3.2; 95\% CI 2.8 to 3.5); AIDS diagnosis age between 30 and 49 years (HR 1.4; 95\% CI 1.2 to 1.5), age over 50 (HR 2.0; $95 \%$ CI 1.7 to 2.3 ); MSM (HR 1.1; 95\% CI 1.1 to 1.2 ) and IDU (HR 1.5 ; $95 \%$ CI 1.3 to 1.6$)$ exposure categories; up to 8 years of schooling (HR 1.4; 95\% CI 1.3 to 1.5) and no schooling (HR 2.1; 95\% CI 1.6 to 2.8); and CD4 count between 350 and 500 cells $/ \mathrm{mm}^{3}$ (HR $1.2 ; 95 \%$ CI 1.1 to 1.2 ) and $<350$ cells $/ \mathrm{mm}^{3}$ (HR 1.3; 95\% CI 1.2 to 1.3 ). Conclusions Increase in AIDS survival and decrease in the mortality rates were observed with HAART. Predictor factors to death were AIDS diagnosis period, age, transmission categories, schooling and CD4 count. The results show the great positive impact of the Brazilian National AIDS Program.

\section{SP3-47 SPATIAL PROXIMITY AND CHILDHOOD HOSPITAL ADMISSIONS IN A DENSELY POPULATED CONURBATION: EVIDENCE FROM HONG KONG'S “CHILDREN OF 1997" BIRTH COHORT}

doi:10.1136/jech.2011.1429760.47

C M Schooling, C Yau, M K Kwok, ${ }^{*}$ B J Cowling, T H Lam, G M Leung. Life course and Lifestyle Epidemiology Group, School of Public Health, Li Ka Shing Faculty of Medicine, The University of Hong Kong, Hong Kong SAR, China

Introduction Physical distance is a barrier to hospital utilisation. In a very densely populated city in China, we examined whether use of public hospitals by children was associated with individual-level residential proximity, and whether these associations varied with type of admission.
Methods The authors used multivariable negative binomial regression in a large, population-representative birth cohort to examine the adjusted associations of proximity to hospitals with Accidents and Emergency services, proxied by distance to the nearest such hospital, with hospital admissions, bed-days and average length of stay from 8 days to 8 years of age.

Results Physical proximity was positively associated with emergency admissions in children (incidence rate ratio (IRR) $1.23,95 \%$ CI 1.11 to 1.35 for $<1 \mathrm{~km}$ compared to $\geq 2 \mathrm{~km}$ ) and bed-days but not with average length of stay, adjusted for age, sex and socio-economic position. However, in a similar comparison there was no such association for other (ie, planned) admissions (IRR 1.04, 95\% CI 0.85 to 1.27).

Conclusion Proximity was associated with hospital use for emergency admissions. Given the societal costs of such use and the risks of iatrogenesis, attention should focus on achieving a more effective use of scarce resources.

\section{SP3-48 ROUTINE MORTALITY AND CAUSE OF DEATH REPORTING AND ANALYSIS SYSTEMS IN SEVEN PACIFIC ISLAND COUNTRIES}

doi:10.1136/jech.2011.1429760.48

${ }^{1} \mathrm{~K}$ Carter, ${ }^{* 1} \mathrm{C}$ Rao, ${ }^{1} \mathrm{~A}$ Lopez, ${ }^{1,2} \mathrm{R}$ Taylor. ${ }^{1}$ School of Population Health, University of Queensland, Herston (Brisbane), Queensland, Australia; ${ }^{2}$ School of Public Health and Community Medicine, University of New South Wales, Sydney, New South Wales, Australia

Introduction Statistics on mortality levels and causes of death are essential for health planning. However, at the end of 2003, only 7 of 27 countries in the Western Pacific Region had data available on causes of death. Routine death reporting systems across seven Pacific Island Countries; Fiji, Kiribati, Nauru, Palau, Solomon Islands, Tonga and Vanuatu, are examined. Strengths and limitations common across national systems are identified, and system characteristics related to data availability and quality.

Methods System assessments included key informant interviews, observation of processes, and document review. Findings were grouped according to a framework that classifies system characteristics according to societal issues, the national administrative environment, administration, technical and ownership issues.

Results Routine reporting of deaths is predominantly managed through civil registration systems or within Health departments. Health reporting systems are critical in supporting the civil registration process. Significantly more information is available than currently used. Legislation on death reporting exists for all islands, but does not necessarily reflect current practices. Significant duplication of data collection and entry exists across all systems. The close interaction between health staff and local communities could provide a good foundation for further improvement in death reporting in these countries. Responsibility, authority and ownership were central to the sustainability of the reporting systems.

Conclusion For Pacific Island Countries to effectively address health challenges there is no substitute for routine mortality and cause of death data collections. Suitable systems exist, but need to be strengthened to improve the completeness and quality of the data available.

SP3-49 THE RELATIONSHIP OF CLASS CLOSURE LENGTH AND THE CHANGE OF ABSENTEES AT ELEMENTARY SCHOOLS IN THE 2009 A/H1N1 INFLUENZA EXPANSION IN JAPAN: THE ANALYSIS IN T CITY, IBARAKI PREFECTURE

doi:10.1136/jech.2011.1429760.49

${ }^{1} \mathrm{~S}$ Yamamoto, ${ }^{*} \mathrm{H}$ Takahashi, ${ }^{3}$ I Wada, ${ }^{4} \mathrm{~A}$ Uda, ${ }^{5} \mathrm{~F}$ Irie. ${ }^{1}$ School of Medicine, School of Medicine and Medical Sciences, University of Tsukuba, Tsukuba, Ibaraki, 